\title{
A NEW METHOD TO ESTIMATE STOCHASTIC VOLATILITY MODELS: A LOG-GARCH APPROACH
}

\author{
Manabu Asai*
}

\begin{abstract}
Changes in asset return variance or volatility over time may be modeled using the GARCH class models or stochastic volatility (SV) models. The log-GARCH models are the logarithmic extension of the GARCH models. The GARCH models are popular and easily estimated. Compared to the GARCH models, the SV models are more general in several respects, but it is well recognized that they are not easy to estimate. In this paper, we derive a log-GARCH representation of a class of SV models, including the ARMA-SV model, and analyze the finite sample properties of a Quasi-Maximum Likelihood (QML) estimator based on the log.GARCH representation. Our Monte Carlo results indicate that their finite sample properties are superior to those of the Generalized Method of Moments estimator and those of the QML estimator based on the Kalman filter; and close to those of the nonlinear filtering maximum likelihood estimator which is a computationally intensive method. We present an empirical example of daily observations on the yen/dollar exchange rate.
\end{abstract}

Key Words and Phrases: log-GARCH models, Quasi-maximum likelihood, Kalman filter, stochastic volatility.

\section{Introduction}

Changes in asset return variance or volatility over time may be modeled using the GARCH models, developed by Engle (1982) and Bollerslev (1986). In GARCH models, such effects are captured by letting the conditional volatility be a function of squares of previous observations and past volatilities. Since the models are formulated in terms of the conditional distribution, the maximum likelihood estimation may be implemented straightforwardly. A wide range of GARCH models have now appeared in the econometric literature; see, for example, a survey by Bollerslev et al. (1995).

An alternative approach is to use an unobserved volatility component model. The logarithm of an unobserved volatility is modeled as a linear stochastic process, such as an autoregression. Models of this kind are called stochastic volatility (SV) models. Their statistical properties are easily obtained from the properties of the process generating the volatility component. Their main disadvantage, however, is that they are difficult to estimate by the maximum likelihood estimation method. Taylor (1986), Melino and Turnbull (1990), and Andersen and Sørensen (1996), used the method of moments (MM) to avoid integration problems associated with evaluating the likelihood directly. Nelson (1988), Harvey et al. (1994) and Ruiz (1994) employed approximate Kalman filtering methods in their quasi maximum likelihood (QML) estimation. The Monte Carlo evidence of Jacquier et al. (1994), however,

Received October, 1997. Revised December, 1997. Accepted February, 1998.

* Doctoral Program in Policy and Planning Sciences, University of Tsukuba, Tsukuba-shi, Ibaraki-ken 305-8573 Japan; masai @ sk.tsukuba.ac.jp. 
implies that $\mathrm{MM}$ and Kalman filtering procedures suffer from poor finite sample performance because they do not depend on the exact likelihood.

When researchers can neglect certain computational costs, there are better alternatives based on the exact likelihood: Danielsson and Richard (1993) and Danielsson (1994a) proposed simulation-based maximum likelihood (SML) procedures; Watanabe (1997) developed nonlinear filtering maximum likelihood (NFML) procedures; Jacquier et al. (1994) suggested a Bayesian inference and used the Markov chain Monte Carlo (MCMC). Although these methods are computationally intensive, experimental results of Jacquier et al. (1994), Danielsson (1994b) and Watanabe (1997), show that these estimators outperform MM and Kalman filter approaches.

The log-GARCH models, proposed by Geweke (1986) and Pantula (1986), are the logarithmic extension of the GARCH models. Their models may be interpreted as a special case of the Exponential GARCH models, originally developed by Nelson (1991). We derive a log-GARCH representation of a class of SV models, including linear regression models with $\operatorname{ARMA}(p, q)$-SV errors. To estimate these SV models, we propose a new QML method via the $\log$-GARCH approach based on either Gaussian or standardized $t$ distribution.

We conduct Monte Carlo experiments to analyze the finite sample property of our method to estimate simple SV models. In parameter estimation, the performance of QML estimator via the $\log$-GARCH approach is better than that of GMM estimators and QML estimators via the Kalman filter and close to that of NFML estimator, which is a computationally intensive method. In volatility estimation using approximate Kalman filtering methods, smoothed estimates with QML estimates via the log-GARCH approach based on standardized $t$ distribution outperform those with GMM estimators and QML estimators via the Kalman filter.

Organization of this paper is as follows: Section 2 shows that a simple SV process in a sequence, say $y_{t}$, can be interpreted as a log-GARCH process in $y_{t}$. Section 3 proposes a QML method via the log-GARCH approach and presents Monte Carlo results. Section 4 reports a brief empirical findings for the yen/ dollar daily exchange rate, and Section 5 concludes the paper.

\section{Stochastic Volatility Processes}

A simple stationary SV model is given by

$$
\begin{aligned}
& y_{t}=\sqrt{h_{t}} \eta_{t}, \quad \eta_{t} \sim \operatorname{NID}(0,1), \\
& \ln h_{t}=\gamma+\phi \ln h_{t-1}+\sigma_{\nu} \nu_{t}, \quad \nu_{t} \sim \operatorname{NID}(0,1),
\end{aligned}
$$

where $\eta_{t}$ is generated independently of $\nu_{t}$ and $|\phi|<1$. Working with logarithms ensures that $h_{t}$ is always positive. As noted in the previous section, MM and Kalman filter approaches are more easy to conduct than the computer intensive methods: SML, NFML and Bayesian MCMC. Some experimental results, however, imply that computer intensive estimators outperform MM and Kalman filter approaches.

As pointed out by Harvey et al. (1994) and Shephard (1996), the simple SV 
process in $y_{t}$ is equivalent to an $\operatorname{ARMA}(1,1)$ process $\ln y_{t}^{2}$ with a skewed non-Gaussian noise. In this subsection, we first derive the parameters of the $\operatorname{ARMA}(1,1)$ representation using the results of Hamilton (1994, chapter 4). We next show that the simple SV process in $y_{t}$ is equivalent to a $\log$-GARCH $(1,1)$ process in $y_{t}$ with a symmetric non-Gaussian noise.

In the simple SV model (2.1), transforming $y_{t}$ by taking logarithms of the squares and concentrating out $h_{t}$, we obtain

$$
\ln y_{t}^{2}=\phi \ln y_{t-1}^{2}+\gamma+\sigma_{\nu} \nu_{t}+\ln \eta_{t}^{2}-\phi \ln \eta_{t-1}^{2} .
$$

The mean and variance of $\ln \eta_{t}^{2}$ are known to be

$$
c_{\eta} \equiv E\left[\ln \eta_{t}^{2}\right]=\left[\phi\left(\frac{1}{2}\right)-\ln \left(\frac{1}{2}\right)\right] \simeq-1.27036, \quad \operatorname{Var}\left[\ln \eta_{t}^{2}\right]=\phi^{\prime}\left(\frac{1}{2}\right)=\frac{\pi^{2}}{2},
$$

where $\psi(\cdot)$ is the Digamma function and $\psi^{\prime}(\cdot)$ is the Trigamma function, defined as

$$
\psi(z) \equiv d[\ln \Gamma(z)] / d z=\Gamma^{\prime}(z) / \Gamma(z), \quad \psi^{\prime}(z) \equiv d \psi(z) / d z=d^{2}[\ln \Gamma(z)] / d^{2} z,
$$

see Abramovitz and Stegun (1970, p. 943).

Equation (2.2) can be rewritten as

$$
\begin{gathered}
\ln y_{t}^{2}=(1-\phi) c_{\eta}+\gamma+\phi \ln y_{t-1}^{2}+\sigma_{\nu} \nu_{t}+\left(\ln \eta_{t}^{2}-c_{\eta}\right)-\phi\left(\ln \eta_{t-1}^{2}-c_{\eta}\right), \\
\left(\ln \eta_{t}^{2}-c_{\eta}\right) \sim \operatorname{iid}\left(0, \pi^{2} / 2\right),
\end{gathered}
$$

where iid $\left(0, \pi^{2} / 2\right)$ denotes an independently identically distributed process with mean zero and variance $\pi^{2} / 2$. Let us note that a white noise process variable combined with an MA(1) variable reduces to an MA(1) process; see Hamilton (1994, chapter 4, pp. 102-106). Using this fact, the last three terms of equation (2.3) becomes an MA(1) process,

$$
\sigma_{\nu} \nu_{t}+\left(\ln \eta_{t}^{2}-c_{\eta}\right)-\phi\left(\ln \eta_{t-1}^{2}-c_{\eta}\right)=\varepsilon_{t}-\theta \varepsilon_{t-1}, \quad \varepsilon_{t} \sim \mathrm{WN}\left(0, \sigma^{2}\right),
$$

where $\mathrm{WN}\left(0, \sigma^{2}\right)$ denotes a white noise process with mean zero and variance $\sigma^{2}$, and

$$
\sigma^{2}=\frac{\pi^{2} \phi}{2 \theta}, \theta=\frac{1}{2 \phi}\left[1+\phi^{2}+\frac{2 \sigma_{\nu}^{2}}{\pi^{2}}-\sqrt{\left(1-\phi^{2}+\frac{2 \sigma_{\nu}^{2}}{\pi^{2}}\right)^{2}+\frac{8 \phi^{2} \sigma_{\nu}^{2}}{\pi^{2}}}\right] .
$$

The other solution of the quadratic equation for $\theta$ does not satisfy the invertibility condition. Note that equation (2.5) implies $\phi \theta>0$ and $0<|\theta|<|\phi|<1$.

As a result, the model reduces to an $\operatorname{ARMA}(1,1)$ process in $\ln y_{t}^{2}$ :

$$
\ln y_{t}^{2}=(1-\phi)_{\eta}+\gamma+\phi \ln y_{t-1}^{2}+\varepsilon_{t}-\theta \varepsilon_{t-1}, \quad \varepsilon_{t} \sim \mathrm{WN}\left(0, \sigma^{2}\right),
$$

where $\varepsilon_{t}$ is a skewed and leptokurtic white noise; see Appendix A.

Next, we show a simple SV process in $y_{t}$ is equivalent to a $\log$-GARCH $(1,1)$ process in $y_{t}$ with a symmetric non-Gaussian noise. Define a standardized process $z_{t}$ and a positive predetermined variable $\sigma_{t}$ by 


$$
\begin{aligned}
& \text { (2.7) } \quad z_{i} \equiv \frac{1}{c_{z}}\left(\eta_{t} \prod_{i=1}^{\infty}\left|\eta_{t-i}\right|^{(\theta-\phi) \theta^{\theta-1}}\right) \exp \left[-\frac{c_{\eta}(1-\phi)}{2(1-\theta)}+\frac{\sigma_{\nu}}{2} \nu_{t}+\frac{\sigma_{\nu}}{2} \sum_{i=1}^{\infty} \theta^{i} \nu_{t-i}\right], \\
& \text { (2.8) } \ln \sigma_{t}^{2} \equiv \ln c_{z}^{2}+\frac{1}{1-\theta}\left(\gamma+(1-\phi) c_{\eta}\right)+(\phi-\theta)(1-\theta L)^{-1} \ln y_{t-1}^{2},
\end{aligned}
$$

where

$$
\begin{aligned}
C_{z}= & \exp \left[\frac{\sigma_{\nu}^{2}}{4\left(1-\theta^{2}\right)}-\frac{c_{\eta}}{2}-\frac{(\theta-\phi) \theta}{2(1-\theta)} \psi\left(\frac{1}{2}\right)\right. \\
& \left.+\frac{1}{2} \sum_{i=1}^{\infty}\left[\ln \Gamma\left((\theta-\phi) \theta^{i}+\frac{1}{2}\right)-\ln \Gamma\left(\frac{1}{2}\right)\right]\right],
\end{aligned}
$$

and $L$ denotes the lag operator. $z_{t}$ is a weak stationary process that has, at least, up to fourth order moments if $|\phi|<1$ and $\sigma_{\nu}>0$; see Appendix B. $\sigma_{t}$ is measurable with respect to the time $t-1$ information set.

Rewriting $\varepsilon_{t}$ in terms of $z_{t}$ or $\sigma_{t}$ as

$$
\varepsilon_{t}=\ln z_{t}^{2}-\ln c_{z}^{2}=\ln y_{t}^{2}-\ln \sigma_{t}^{2}-\ln c_{z}^{2},
$$

and substituting equation (2.9) to (2.6), we obtain an equivalent representation of the $\log \mathrm{AR}(1) \mathrm{SV}$ process,

(2.10) $y_{t}=\sigma_{t} z_{t}, \quad z_{t} \sim \mathrm{WN}(0,1)$,

$$
\ln \sigma_{t}^{2}=\left[\gamma+(1-\phi) c_{\eta}+(1-\theta) \ln c_{z}^{2}\right]+(\phi-\theta) \ln y_{t-1}^{2}+\theta \ln \sigma_{t-1}^{2} .
$$

Note that $z_{t}$ is serially uncorrelated with mean zero and variance one. Therefore the log-AR(1) SV process in $y_{t}$ can be interpreted as a log-GARCH $(1,1)$ process in $y_{t}$ which has a heavy-tailed and symmetric conditional distribution.

Reparameterizing $\left(\gamma, \phi, \sigma_{\nu}\right)$ as

$$
\begin{aligned}
& \alpha_{0} \equiv \gamma+(1-\phi) c_{\eta}+(1-\theta) \ln c_{z}^{2}, \\
& \alpha_{1} \equiv \phi-\theta, \\
& \beta_{1} \equiv \theta,
\end{aligned}
$$

we have

$$
\begin{aligned}
& y_{t}=\sigma_{t} z_{t}, \\
& \ln \sigma_{t}^{2}=\alpha_{0}+\alpha_{1} \ln y_{t-1}^{2}+\beta_{1} \ln \sigma_{t-1}^{2},
\end{aligned}
$$

where $\left|\alpha_{1}+\beta_{1}\right|<1$ and $\left(\alpha_{1}+\beta_{1}\right) \beta_{1}>0$. The inverse transformations for this mapping are

$$
\begin{aligned}
\gamma= & \alpha_{0}+\alpha_{1} c_{\eta}+\frac{\alpha_{1}\left(1-\left(\alpha_{1}+\beta_{1}\right) \beta_{1}\right) \pi^{2}}{4 \beta_{1}\left(1+\beta_{1}\right)}+\alpha_{1} \beta_{1} \psi\left(\frac{1}{2}\right) \\
& +\left(1-\beta_{1}\right) \sum_{i=1}^{\infty}\left[\ln \Gamma\left(\frac{1}{2}-\alpha_{1} \beta_{1}^{i}\right)-\ln \Gamma\left(\frac{1}{2}\right)\right], \\
\phi= & \alpha_{1}+\beta_{1}, \\
\sigma_{\nu}= & \sqrt{\frac{(\phi-\theta)(1-\theta \phi) \pi^{2}}{2 \theta}}=\sqrt{\frac{\alpha_{1}\left(1-\left(\alpha_{1}+\beta_{1}\right) \beta_{1}\right) \pi^{2}}{2 \beta_{1}}} .
\end{aligned}
$$


Note that

$$
\begin{aligned}
\theta= & \beta_{1}, \\
c_{2}^{2}= & \exp \left[\frac{\alpha_{1}\left(1-\left(\alpha_{1}+\beta_{1}\right) \beta_{1}\right) \pi^{2}}{4 \beta_{1}\left(1-\beta_{1}^{2}\right)}-c_{\eta}+\frac{\alpha_{1} \beta_{1}}{1-\beta_{1}} \psi\left(\frac{1}{2}\right)\right. \\
& \left.+\sum_{i=1}^{\infty}\left[\ln \Gamma\left(\frac{1}{2}-\alpha_{1} \beta_{1}^{i}\right)-\ln \Gamma\left(\frac{1}{2}\right)\right]\right] .
\end{aligned}
$$

We now consider a linear regression model with $\operatorname{ARMA}(p, q)$-SV errors, or simply ARMA-SV model:

$$
\begin{aligned}
& y_{t}=X_{t} \delta+u_{t}, \\
& A(L) u_{t}=B(L) e_{t}, \\
& e_{t}=\sqrt{h_{t}} \eta_{t}, \quad \eta_{t} \sim \operatorname{NID}(0,1), \\
& \ln h_{t}=\gamma+\phi \ln h_{t-1}+\sigma_{\nu} \nu_{t}, \quad \nu_{t} \sim \operatorname{NID}(0,1),
\end{aligned}
$$

where $\eta_{t}$ is generated independently of $\nu_{t}, X_{t}$ is a $1 \times k$ vector, $\delta$ is a $k \times 1$ parameter vector,

$$
\begin{aligned}
& A(L)=1-a_{1} L-\cdots-a_{p} L^{p}, \\
& B(L)=1-b_{1} L-\cdots-b_{q} L^{q},
\end{aligned}
$$

and $L$ is the lag operator. This ARMA-SV model is a straightforward extension if we take account of the empirical results that many asset return series may be expressed as ARMA processes.

The ARMA-SV model of (2.12) - (2.15) can be interpreted as an ARMA$\log -\operatorname{GARCH}(1,1)$ model in a similar fashion to the simple SV model (1):

$$
\begin{aligned}
& y_{t}=X_{t} \delta+u_{t}, \\
& A(L) u_{t}=B(L) e_{t}, \\
& e_{t}=\sigma_{t} z_{t}, \quad z_{t} \sim \mathrm{WN}(0,1), \\
& \ln \sigma_{t}^{2}=\alpha_{0}+\alpha_{1} \ln e_{t-1}^{2}+\beta_{1} \ln \sigma_{t-1}^{2},
\end{aligned}
$$

where the definitions of the transformed parameters $\left(\alpha_{0}, \alpha_{1}, \beta_{1}\right)$ are the same as in the simple SV case. $z_{t}$ has the heavy-tailed and symmetric conditional distribution.

\section{Econometric Methodology}

\subsection{A New QML Estimation Method}

Under a set of mild regularity conditions, the quasi-maximum likelihood estimator proposed by White (1982) is consistent and asymptotically normal. Lee and Hansen (1994) established the consistency and asymptotic normality properties of the quasi-maximum likelihood estimator of the $\operatorname{GARCH}(1,1)$ and $\operatorname{IGARCH}(1,1)$ models assuming that the standardized variable $z_{t}$ is stationary and ergodic with a bounded fourth conditional moment. Unfortunately, as with other ARCH models including EGARCH models, a satisfactory asymptotic theory for the log-GARCH is as yet unavailable. 
In the remainder of this paper, we assume that the quasi-maximum likelihood estimator is consistent and asymptotically normal ${ }^{11}$. The asymptotic distribution for the QML estimator of the parameter vector $\omega \equiv\left(a_{0}, a_{1}, b_{1}\right)^{\prime}$ takes the form

$$
\sqrt{T}\left(\hat{\omega}_{\text {QMLE }}-\omega_{0}\right) \stackrel{a}{\sim} N\left(0, A_{0}^{-1} B_{0} A_{0}^{-1}\right),
$$

where $A_{0}$ is the information matrix evaluated at the true parameter vector, and $B_{0}$ is the expected value of the outer product of the gradients evaluated at the true parameters. Hence, the asymptotic distribution for the QML estimator of $f(\omega)=\left(\gamma, \phi, \sigma_{\nu}\right)^{\prime}$ takes the form

$$
\sqrt{T}\left(f\left(\hat{\omega}_{\text {QMLE }}\right)-f\left(\omega_{0}\right)\right) \stackrel{a}{\sim} N\left(0,\left(\frac{\partial f\left(\omega_{0}\right)}{\partial \omega^{\prime}}\right) A_{0}^{-1} B_{0} A_{0}^{-1}\left(\frac{\partial f\left(\omega_{0}\right)}{\partial \omega^{\prime}}\right)^{\prime}\right)
$$

As in Melino and Turnbull (1990), Harvey et al. (1994) and Ruiz (1994), we can obtain smoothed estimates of $\ln h_{t}$ by applying the approximate Kalman filter, described in Anderson and Moore (1979, chapter 8). The smoothed estimates of $h_{t}$ may be converted from the smoothed estimates of log-volatility ln $h_{t}$ using standard properties of lognormal distribution ${ }^{2}$.

\subsection{Monte Carlo Experiments}

In this subsection, we investigate the finite sample property of the QML estimator via log-GARCH approach for the simple SV model. Jacquier et al. (1994) have surveyed the literature and considered parameters designs which seem to be adequate in empirical studies. Following Andersen and Sørensen (1996) and Watanabe (1997), we focus on the following three parameter settings of Jacquier et al. (1994):

$$
\begin{aligned}
\left(\gamma, \phi, \sigma_{\nu}\right) & =(-0.736,0.90,0.363) \\
& =(-0.368,0.95,0.260) \\
& =(-0.147,0.98,0.166) .
\end{aligned}
$$

While Jacquier et al. (1994) consider sample size of $T=500$ in the majority of their experiments, we use $T=2000$, which is not uncommon in studies using daily data. The number of replications is 1000 .

To construct the log-GARCH quasi-log-likelihood, we treat $z_{t}$ in equations (2.10) as (a) a standard normal variable, and (b) a standardized $t$ variable favored by Bollerslev (1987), among others. As noted in the previous section, $z_{t}$ has a heavy-tailed and symmetric distribution, and thus the standardized $t$ distribution is more appealing than the normal distribution. When the distribution of $z_{t}$ conditioned on the information up to time $t-1$ assumed to be

1) This is the usual practice in papers that use ARCH models. See, e.g., Bollerslev et al. (1995).

2) There are two more accurate computationally intensive smoothing procedures. Jacquire $e t$ al. (1994) used a method based on Bayesian Markov chain Monte Carlo. Watanabe (1997) proposed a smoothing procedure based on a nonlinear filtering method. Watanabe's (1997) Monte Carlo results indicate that his nonlinear smoothing conditional on NFML estimates produces more efficient volatility estimates than the approximate Kalman filtering condi. tional on true parameters. 
standardized $t$ with degrees of freedom $\lambda$, the conditional quasi-distribution of $y_{t}$ is standardized $t$ with mean zero and variance $\sigma_{t}^{2}$, i.e.,

$$
f\left(y_{t}\right)=\Gamma\left(\frac{\lambda+1}{2}\right) \Gamma\left(\frac{\lambda}{2}\right)^{-1}\left[(\lambda-2) \sigma_{t}^{2}\right]^{-1 / 2}\left[1+\frac{y_{t}^{2}}{(\lambda-2) \sigma_{t}^{2}}\right]^{-(\lambda+1) / 2}(\lambda>2),
$$

where $f\left(y_{t}\right)$ denotes the conditional quasi-density function of $y_{t}$. Analytic derivatives of the log-GARCH quasi-log-likelihood are used instead of numerical differentiation ${ }^{3}$.

Table 1 shows the mean and the root mean squared error (RMSE) of various estimators. Results of GMM, SML and Bayesian MCMC estimators are respectively obtained from Table 5 and Table 9 of Andersen and Sørensen (1996), Table 2 of Danielsson (1994b) and Table 9 of Jacquier et al. (1994). Results of QML estimators via the Kalman filter approach and NFML estimators are obtained from Table 1 of Watanabe (1997).

QML estimate biases via the log-GARCH approach based on the normal or

Table 1. Comparison of QML estimator via log-GARCH approach and other estimators, $T=2000$ (1000 replications)

\begin{tabular}{c|ccc|ccc|ccc}
\hline \multirow{2}{*}{ Method } & $\gamma$ & $\phi$ & $\sigma_{\nu}$ & $\gamma$ & $\phi$ & $\sigma_{\nu}$ & $\gamma$ & $\phi$ & $\sigma_{\nu}$ \\
\cline { 2 - 9 } & -.736 & .900 & .363 & -.368 & .950 & .260 & -.147 & .980 & .166 \\
\hline LGARCH-n & -.766 & .895 & .351 & -.393 & .946 & .253 & -.175 & .976 & .167 \\
& $(.269)$ & $(.036)$ & $(.074)$ & $(.147)$ & $(.020)$ & $(.048)$ & $(.080)$ & $(.011)$ & $(.032)$ \\
\hline LGARCH-t & -.773 & .894 & .363 & -.396 & .954 & .261 & -.175 & .976 & .170 \\
& $(.234)$ & $(.031)$ & $(.066)$ & $(.134)$ & $(.018)$ & $(.044)$ & $(.074)$ & $(.010)$ & $(.030)$ \\
\hline GMM & -.592 & .920 & .279 & -.286 & .961 & .190 & -.140 & .981 & .125 \\
& $(.311)$ & $(.042)$ & $(.115)$ & $(.197)$ & $(.027)$ & $(.099)$ & $(.112)$ & $(.015)$ & $(.068)$ \\
\hline Kalman filter & -.837 & .887 & .383 & -.419 & .943 & .271 & -.182 & .975 & .174 \\
& $(.369)$ & $(.050)$ & $(.095)$ & $(.187)$ & $(.025)$ & $(.061)$ & $(.087)$ & $(.012)$ & $(.037)$ \\
\hline NFML & -.812 & .890 & .406 & -.426 & .942 & .294 & -.194 & .974 & .197 \\
$N=25$ & $(.199)$ & $(.027)$ & $(.068)$ & $(.124)$ & $(.017)$ & $(.052)$ & $(.083)$ & $(.011)$ & $(.043)$ \\
\hline NFML & -.776 & .895 & .368 & -.406 & .945 & .264 & -.178 & .976 & .169 \\
$N=50$ & $(.168)$ & $(.023)$ & $(.041)$ & $(.106)$ & $(.014)$ & $(.032)$ & $(.067)$ & $(.009)$ & $(.024)$ \\
\hline SML & -.721 & .902 & .359 & & & & & & \\
& $(.15)$ & $(.02)$ & $(.039)$ & & & Not Available & & \\
\hline Bayesian & -.762 & .896 & .359 & & & & & & \\
MCMC & $(.15)$ & $(.02)$ & $(.034)$ & & & & & & \\
\hline
\end{tabular}

Note: 'LGARCH-n' donotes the log-GARCH approach based on the normal distribution. 'LGARCH- $t$ ' denotes the log-GARCH approach based on the standardized $t$ distribution. Results of GMM, SML and Bayesian MCMC estimators are respectively obtained from Table 5 and Table 9 of Andersen and Sørensen (1996), Table 2 of Danielsson (1994b) and Table 9 of Jacquier et al. (1994). Results of QML estimators via the Kalman filter approach and NFML estimators are obtained from Table 1 of Watanabe (1997). $N$ is the number of nodes used in NFML. The table shows the mean and RMSE (in parentheses).

3) Fiorentini, Calzolari, and Panattoni (1996) establish a computational advantage of analytic derivatives of the GARCH log-likelihood over a numerical differentiation. The advantage is large especially when second-order derivatives are involved in the computation. 
the standardized $t$ distribution are smaller than those of GMM estimates, QML estimates via the Kalman filter approach and NFML estimates when $N=25$, where $N$ is the number of nodes used in NFML. These biases resemble NFML estimates when $N=50$, SML and Bayesian MCMC estimates. The RMSE of QML estimates via the log-GARCH approach based on the normal or the standardized $t$ distribution are smaller than those of GMM estimates, QML estimates via the Kalman filter approach, and larger than those of NFML estimates when $N=50$, SML and Bayesian MCMC estimates.

These results imply that, 1) QML estimators via the log-GARCH approach outperform GMM estimators and QML estimators via the Kalman filter approach, and 2) the performance of estimators based on computationally intensive methods except NFML estimators when $N=25$ are mainly superior to that of QML estimators via the log-GARCH approach.

\subsection{Smoothing Performance}

We compare the smoothing performance of the approximate Kalman filtering conditional on the true parameters, and QML estimates via the logGARCH approach based on the normal and those based on the standardized $t$ distribution. Following Jacquier et al. (1994) and Watanabe (1997), we compute the grand RMSE,

$$
\mathrm{RMSE}=\sqrt{\frac{1}{1000(T-199)} \sum_{i=1}^{1000} \sum_{t=100}^{T-100}\left(h_{i, t}-\hat{h}_{i, t}\right)^{2}},
$$

where $T=2000, h_{i, t}$ is the volatility simulated at period $t$ in the $i$ th simulation, and $\hat{h}_{i, t}$ is its smoothed estimate. The smoothed estimates of $h_{i, t}$ may be converted from the smoothed estimates of $\log$-volatility $\ln h_{i, t}$ using standard properties of lognormal distribution.

Table 2 presents the RMSE of smoothed estimates of volatility. The

Table 2. RMSE smoothing performance of approximate Kalman filtering, $T=2000$ (1000 replications)

\begin{tabular}{lccc}
\hline & \multicolumn{3}{c}{$\phi$} \\
\cline { 2 - 4 } Method & .90 & .95 & .98 \\
\hline True parameters & 6.856 & 6.099 & 5.127 \\
LGARCH-n & 6.923 & 6.127 & 5.133 \\
LGARCH-t & 6.884 & 6.114 & 5.125 \\
\hline True parameters & 6.85 & 6.08 & 5.24 \\
KF & 6.92 & 6.13 & 5.30 \\
\hline
\end{tabular}

Note: The second half of table 2 are obtained from Table 2 of Watanabe (1997). 'LGARCH-n' denotes the log-GARCH approach based on the normal distribution. 'LGARCH-t' denotes the $\log -\mathrm{GARCH}$ approach based on the standardized $t$ distribution. 'KF' denotes the Kalman filter approach. RMSE $\times 10000$ is displayed. 
second half of table 2 is obtained from Table 2 of Watanabe (1997). Smoothing solutions with QML estimates via the log-GARCH approach based on the normal distribution resemble those of the Kalman filtering method. Smoothing solutions with QML estimates via the log-GARCH approach based on the standardized $t$ distribution dominates these two results. Since Jacquier et al. (1994) reported that RMSE of smoothing solutions conditional on QML estimates via the Kalman filter are smaller than those with GMM estimates, our results show that smoothing solutions via the log-GARCH approach based on the standardized $t$ distribution dominates those with GMM estimates or QML estimates via the Kalman filter.

\section{Empirical Example: Daily Exchange Rates}

The data consist of daily yen/dollar close exchange rates, $S_{t}$, from the Tokyo interbank market ${ }^{4}$. There are total of 1484 daily observations, from January 4, 1991 to December 30, 1996. We calculate the rate of change, $r_{t}$, by taking the logarithmic difference between the close of two successive trading days.

Table 3 provides some summary statistics of the data. The data are unimodal and approximately symmetric, with higher peaks and fatter tail than the Gaussian distribution.

We consider the following MA(1)-SV model:

$$
\begin{aligned}
& r_{t}=\delta_{0}+\delta_{1} W_{t}+e_{t}-b_{1} e_{t-1}, \\
& e_{t}=\sqrt{h_{t}} \eta_{t} \quad \eta_{t} \sim \operatorname{NID}(0,1), \\
& \ln h_{t}=\gamma_{0}+\gamma_{1} W_{t}-\gamma_{1} \phi W_{t-1}+\phi \ln h_{t-1}+\sigma_{\nu} \nu_{t}, \quad \nu_{t} \sim \operatorname{NID}(0,1),
\end{aligned}
$$

where $W_{t}$ denotes a weekend dummy equal to one following a closure of the market. The MA(1) term is included to take account of the weak serial dependence in the mean. Following Baillie and Bollerslev (1989), the weekend dummy is entered in variance to allow for an impulse effect.

The above MA(1)-SV model in $y_{t}$ may be interpreted as an MA(1)-log$\operatorname{GARCH}(1,1)$ model in $y_{t}$ with the similar discussion to section 2 ,

Table 3. Summary Statistics of log price change $r_{t} \times 100=\ln \left(S_{t} / S_{t-1}\right) \times 100$

\begin{tabular}{cccc}
\hline Mean & Std. dev. & Minimum & Maximum \\
\hline-0.0104 & 0.6434 & -3.6258 & 3.3937 \\
\hline \hline Skewness & Kurtosis & $\begin{array}{c}\text { Studentized } \\
\text { range }\end{array}$ & NOBS \\
\hline $\begin{array}{c}-0.1873 \\
(0.0636)\end{array}$ & $\begin{array}{c}6.5473 \\
(0.0162)\end{array}$ & 10.9094 & 1484 \\
\hline
\end{tabular}

Sample period : 4 January 1991 to 30 December 1996.

Standard errors in parentheses.

Note: The standard errors are computed as follows:

$\sqrt{6 / \mathrm{NOBS}}$ for the coefficient of skewness,

$\sqrt{24 / \text { NOBS }}$ for the coefficient of kurtsis.

4) The data are offered by the Ueda Harlow Co. for academic purposes. 


$$
\begin{aligned}
& r_{t}=\delta_{0}+\delta_{1} W_{t}+e_{t}-b_{1} e_{t-1}, \\
& e_{t}=\sigma_{t} z_{t} \\
& \ln \sigma_{t}^{2}=\alpha_{0}+\gamma_{1} W_{t}-\gamma_{1}\left(\alpha_{1}+\beta_{1}\right) W_{t-1}+\alpha_{1} \ln e_{t-1}^{2}+\beta_{1} \ln \sigma_{t-1}^{2},
\end{aligned}
$$

where

$$
\begin{aligned}
& \alpha_{0} \equiv \gamma_{0}+(1-\phi) c_{\eta}+(1-\theta) \ln c_{z}^{2}, \\
& \alpha_{1} \equiv \phi-\theta, \\
& \beta_{1} \equiv \theta .
\end{aligned}
$$

Table 4 reports the QML estimates based on the normal distribution and the standardized $t$ distribution. The robust QML covariance estimators of Bollerslev and Wooldridge (1992) are used to compute the standard errors. Note that they cannot be used to test whether $\sigma_{\nu}$ is significantly different from zero.

The estimate of $\lambda$ is 4.527 and indicates the standardized $t$ distribution is preferred to the normal distribution. In both cases, the coefficients of weekend dummy in level, $\delta_{1}$, are not significant at the five percent level, and the coefficients in volatility, $\gamma_{1}$, are significant. These results support the empirical findings of Hsieh (1988, 1989) and Baillie and Bollerslev (1989). Estimates of $\phi$ are both significant and indicate strong degree of persistence in volatility. Ruiz (1994), however, found much higher value for $\phi$ and fitted a random walk stochastic volatility model. It should be pointed out that the Ruiz (1994) study was conducted from October 1,1981 to June 28, 1985, while our period was from January 4 1991, to December 30, 1996.

Table 4. MA(1)-SV Models

\begin{tabular}{c|cc}
\hline & LGARCH-n & LGARCH- $t$ \\
\hline$\delta_{0}$ & -0.000128 & -0.000020 \\
& $(0.000004)$ & $(0.000005)$ \\
$\delta_{1}$ & -0.000035 & 0.000026 \\
& $(0.000688)$ & $(0.000778)$ \\
$b_{1}$ & -0.005212 & 0.04527 \\
& $(0.001133)$ & $(0.00113)$ \\
$\gamma_{0}$ & -0.5939 & -0.4243 \\
& $(0.0066)$ & $(0.0029)$ \\
$\gamma_{1}$ & 0.2703 & 0.03245 \\
& $(0.0040)$ & $(0.0198)$ \\
$\phi$ & 0.9418 & 0.9577 \\
& $(0.04481)$ & $(0.0186)$ \\
$\sigma_{\nu}$ & 0.2077 & 0.1613 \\
& $(0.1412)$ & $(0.0590)$ \\
$\lambda$ & - & 4.527 \\
& & $(0.00056)$ \\
quasi-log- & 5432.76 & 6347.28 \\
\hline likelihood & & \\
\hline
\end{tabular}

Note) 'LGARCH-n' denotes the $\log \cdot \mathrm{GARCH}$ approach based on the normal distribution. 'LGARCH-t' denotes the log-GARCH approach based on the standardized $t$ distribution. Standard error is in parenthenes. 


\section{Concluding Remarks}

This paper derived a log-GARCH representation of a class of SV models, including linear regression models with $\operatorname{ARMA}(p, q)$-SV errors. To estimate these SV models, we proposed a new QML method via the $\log -\mathrm{GARCH}$ approach based on either Gaussian or standardized $t$ distribution. We conducted Monte Carlo experiments to analyze the finite sample property of our method to estimate simple SV models. In parameter estimation, the performance of the QML estimator via the log-GARCH approach is better than that of either GMM estimators or QML estimators via the Kalman filter, and close to that of NFML estimators, which is a computationally intensive method. It should be emphasized that both Bayesian MCMC and SML estimators outperform QML estimator via the log-GARCH approach when the results are available, and that since the performance of NFML estimators depends on the number of nodes, increasing the number may provide better performance. In volatility estimation using approximate Kalman filtering methods, smoothed estimates with QML estimates via the log-GARCH approach based on standardized $t$ distribution outperforms both those with GMM estimators as well as QML estimators via the Kalman filter. We present empirical example of daily observations on the yen/dollar exchange rate.

Our method has certain advantages, but they still leave room for extensions. For example, using the generalized $t$ distribution as the conditional distribution in the log-GARCH approach may improve the finite sample performances of QML estimators since the generalized $t$ distribution has two shape parameters while the standardized $t$ distribution contains only one shape parameter. This task awaits further research.

\section{Appendix}

The derivation of the moments of $\varepsilon_{t}$ and $z_{t}$ in this paper is available upon request.

\section{A. $\varepsilon_{t}$ and its Moments}

In terms of $\nu_{t}$ and $\eta_{t}, \varepsilon_{t}$ can be expressed as

$$
\varepsilon_{t}=\sigma_{\nu} \nu_{t}+\sigma_{\nu} \sum_{i=1}^{\infty} \theta^{i} \nu_{t-i}+\left(\ln \eta_{t}^{2}-c_{\eta}\right)+\sum_{i=1}^{\infty}(\theta-\phi) \theta^{i-1}\left(\ln \eta_{t-i}^{2}-c_{\eta}\right) .
$$

Since $|\theta|<|\phi|<1, \sum_{i=0}^{\infty}|\theta|^{i}<\infty$ and $\sum_{i=1}^{\infty}|\theta-\phi||\theta|^{i-1}<\infty$. Therefore $\varepsilon_{t}$ is a weak stationary process. Its moments up to fourth order are given by

$$
\begin{aligned}
& E\left(\varepsilon_{t}\right)=0, \quad E\left(\varepsilon_{t}^{2}\right)=\frac{\pi^{2} \phi}{2 \theta}, \\
& \frac{E\left(\varepsilon_{t}^{3}\right)}{\left[E\left(\varepsilon_{t}^{2}\right)\right]^{3 / 2}}=\frac{1}{\pi^{3}}\left[1+\frac{(\theta-\phi)^{3}}{1-\theta^{3}}\right]\left(\frac{2 \theta}{\phi}\right)^{3 / 2} \psi^{(2)}\left(\frac{1}{2}\right), \\
& \frac{E\left(\varepsilon_{t}^{4}\right)}{\left[E\left(\varepsilon_{t}^{2}\right)\right]^{2}}=\frac{4 \theta^{2}}{\phi^{2}}\left[1+\frac{(\theta-\phi)^{4}}{1-\theta^{4}}\right]+3, \\
& E\left(\varepsilon_{t} \varepsilon_{t-j}\right)=0, \text { for } j>1 .
\end{aligned}
$$




\section{B. $z_{t}$ and its Moments}

First we show that $c_{z}<\infty$, that is

$$
\Gamma\left((\theta-\phi) \theta^{i}+\frac{1}{2}\right)>0 \quad \text { and } \sum_{i=1}^{\infty}\left[\ln \Gamma\left((\theta-\phi) \theta^{i}+\frac{1}{2}\right)-\ln \Gamma\left(\frac{1}{2}\right)\right]<\infty \text {. }
$$

Since $\phi$ and $\theta$ always have the same sign and $0<|\theta|<|\phi|<1$,

$$
0<(\phi-\theta) \theta=\frac{\phi^{2}}{4}-\frac{1}{4 \phi^{2}}\left[1+\frac{2 \sigma_{\nu}^{2}}{\pi^{2}}-\sqrt{\left(1-\phi^{2}+\frac{2 \sigma_{\nu}^{2}}{\pi^{2}}\right)^{2}+\frac{8 \phi^{2} \sigma_{\nu}^{2}}{\pi^{2}}}\right]^{2} \leq \frac{\phi^{2}}{4}<\frac{1}{4} .
$$

(i) If $\theta>0$, then $0<(\phi-\theta) \theta^{i}<(\phi-\theta) \theta<1 / 4$.

(ii) If $\theta<0$ and $i$ is odd, $i=2 j+1$, then $0<(\phi-\theta) \theta \cdot \theta^{2 j}<1 / 4$ since $|\theta|<1$.

(iii) If $\theta<0$ and $i$ is even, $i=2 j$, then $(\theta-\phi) \theta^{2 j}>0$.

Therefore $(\theta-\phi) \theta^{i}+1 / 2>0$, and thus $\Gamma\left((\theta-\phi) \theta^{i}+1 / 2\right)>0$.

Define a sequence,

$$
s_{i} \equiv \ln \Gamma\left((\theta-\phi) \theta^{i}+\frac{1}{2}\right)-\ln \Gamma\left(\frac{1}{2}\right) .
$$

By the Taylor expansion, $s_{i}$ can be expressed as

$$
s_{i}=(\theta-\phi) \theta^{i} \psi\left(\frac{1}{2}\right)+o\left(\theta^{i}\right) .
$$

Note that $b_{i}=o\left(a_{i}\right)$ implies $\lim _{i-\infty} b_{i} / a_{i}=0$, and that the Digamma function, $\phi(\cdot)$, is the first derivative of $\ln \Gamma(\cdot)$. Thus

$$
\begin{aligned}
\frac{s_{i+1}}{s_{i}} & =\frac{(\theta-\phi) \theta^{i+1} \psi(1 / 2)+o\left(\theta^{i}\right)}{(\theta-\phi) \theta^{i} \psi(1 / 2)+o\left(\theta^{i}\right)} \\
& =\frac{\theta \psi(1 / 2)+o(1)}{\psi(1 / 2)+o(1)},
\end{aligned}
$$

and $\lim _{i-\infty} s_{i+1} / s_{i}=\theta$. Since there exist a positive constant $K$ such that $\left|s_{i+1} / s_{i}\right|$ $<K<1$ for a sufficiently large $i, \sum_{i=1}^{\infty} s_{i}$ converges absolutely. We have shown that $c_{z}<\infty$.

Second we show the weak stationarity of $z_{t} . \quad z_{t}$ can be rewritten by

$$
z_{t}=\frac{\eta_{t}}{c_{z}} \exp \left[\sum_{i=1}^{\infty}(\theta-\phi) \theta^{i-1}\left|\eta_{t-i}\right|-\frac{c_{\eta}(1-\phi)}{2(1-\theta)}+\frac{\sigma_{\nu}}{2} \nu_{t}+\frac{\sigma_{\nu}}{2} \sum_{i=1}^{\infty} \theta^{i} \nu_{t-i}\right] .
$$

Therefore $z_{t}$ is a weak stationary process since $\sum_{i=1}^{\infty}|\theta-\phi||\theta|^{i-1}<\infty$ and $\sum_{i=0}^{\infty}$ $|\theta|^{i}<\infty$.

The moments of $z_{t}$ are given by

$$
\begin{aligned}
& E\left(z_{t}\right)=0, \quad E\left(z_{t}^{2}\right)=1, \quad E\left(z_{t}^{3}\right)=0, \\
& E\left(z_{t}^{4}\right)= \frac{3}{c_{2}^{4}} \exp \left[\frac{2 \sigma_{\nu}^{2}}{1-\theta^{2}}-2 c_{\eta}-\frac{2(\theta-\phi) \theta}{1-\theta} \psi\left(\frac{1}{2}\right)\right. \\
&\left.+\sum_{i=1}^{\infty}\left[\ln \Gamma\left(2(\theta-\phi) \theta^{i}+\frac{1}{2}\right)-\ln \Gamma\left(\frac{1}{2}\right)\right]\right], \\
& E\left(z_{t} z_{t-j}\right)=0, \quad \text { for } j>1 .
\end{aligned}
$$


Similar way to show $c_{z}<\infty$ reveal that $E\left(z_{t}^{4}\right)<\infty$ if $|\phi|<1$ and $\sigma_{\nu}>0$.

\section{Acknowledgemant}

The author is grateful to the anonymous referee, Professors Tsunemasa Shiba of the University of Tsukuba, Yoshihisa Baba of Soka University, and Toshiaki Watanabe of Tokyo Metropolitan University for many useful suggestions and comments.

\section{REFERENCES}

[1] Abramovitz, M. and Stegun, N. (1970). Handbook of Mathematical Functions, Dover Publications, NY.

[2] Andersen, T. G. and Sørensen, B. E. (1996). GMM estimation of a stochastic volatility model: a Monte Carlo study, Journal of Business and Economic Statistics, 14, 328-353.

[ 3 ] Anderson, B. D. O. and Moore, J. B. (1979). Optimal Filtering, Prentice-Hall, NJ.

[4] Baillie, R. T. and Bollerslev, T. (1989). The message in daily exchange rates: a conditional-variance tail, Journal of Business and Economic Statistics, 7, 297-305.

[5] Bollerslev, T. (1986). Generalized autoregressive conditional heteroskedasticity, Journal of Econometrics, 31, 307-327.

[6] Bollerslev, T. (1987). A conditional heteroskedastic time series model for speculative prices and rate of return, Review of Economics and Statistics, 69, 542-547.

[ 7 ] Bollerslev, T., Engle, R. F. and Nelson, D. B. (1995). ARCH models, In The Handbook of Econometrics, 4, (R. F. Engle and D. McFadden, eds.), North-Holland, Amsterdam, 29593038.

[ 8 ] Bollerslev, T. and Wooldridge, J. M. (1992). Quasi maximum likelihood estimation and inference in dynamic models with time varying covariances, Econometric Reviews, 11, 143172.

[9] Danielsson, J. (1994a). Stochastic volatility in asset prices: estimation with simulated maximum likelihood, Journal of Econometrics, 64, 375-400.

[10] Danielsson, J. (1994b). Bayesian analysis of stochastic volatilities: comment, Journal of Business and Economic Statistics, 12, 393-395.

[11] Danielsson, J. and Richard, J. F. (1993). Quadratic acceleration for Monte Carlo likelihood evaluation, Journal of Applied Econometrics, 8, 153-174.

[12] Engle, R. F. (1982). Autoregressive conditional heteroskedasticity with estimates of the variance of U. K. inflation, Econometrica, 50, 987-1008.

[13] Fiorentini, G., Calzolari, G. and Panattoni, L. (1996). Analytic derivatives and the computation of GARCH estimates, Journal of Applied Econometrics, 11, 399-417.

[14] Geweke, J. (1986). Modeling the persistence of conditional variances: a comment, Econometric Reviews, 5, 57-61.

[15] Hamilton, J. D. (1994). Time Series Analysis, Princeton University Press, NJ.

[16] Harvey, A. C. (1993). Time Series Models (2nd ED.), MIT Press, Cambridge, MA.

[17] Harvey, A. C., Ruiz, E. and Shephard, N. (1994). Multivariate stochastic variance models, Review of Economic Studies, 61, 247-264.

[18] Hsieh, D. A. (1988). The statistical properties of daily foreign exchange rates: 1974-1983, Journal of International Economics, 24, 129-145.

[19] Hsieh, D. A. (1989). Modeling heteroskedasticity in daily foreign-exchange rates, Journal of Business and Economic Statistics, 7, 307-317.

[20] Jacquier, E., Polson, N. G. and Rossi, P. E. (1994). Bayesian analysis of stochastic volatilities, Journal of Business and Economic Statistics, 12, 371-389.

[21] Lee, S. W. and Hansen, B. E. (1994). Asymptotic theory for the $\operatorname{GARCH}(1,1)$ quasimaximum likelihood estimator, Econometric Theory, 10, 29-52.

[22] Melino, A. and Turnbull, S. M. (1990). Pricing foreign currency options with stochastic volatility, Journal of Econometrics, 45, 239-265.

[23] Nelson, D. B. (1988). The time-series behaviour of stock market volatility and returns, Unpublished Ph.D. dissertation, MIT, Cambridge, MA.

[24] Nelson, D. B. (1991). Conditional heteroskedasticity in asset returns: a new approach, Econometrica, 59, 347-370. 
[25] Pantula, S. G. (1986). Modeling the persistence of conditional variances: a comment, Econometric Reviews, 5, 79-97.

[26] Ruiz, E. (1994). Quasi-maximum likelihood estimation of stochastic volatility models, Journal of Econometrics, 63, 289-306.

[27] Shephard, N. (1996). Statistical aspects of ARCH and stochastic volatility, In Time Series Models in econometrics, finance and other fields, (D. R. Cox, D. V. Hinkley and O. E. Barndorff-Nielsen, eds.), Chapman \& Hall, 1-67.

[28] Taylor, S. J. (1986). Modeling Financial Time Series, John Wiley, Chichester.

[29] Watanabe, T. (1997). A nonlinear filtering approach to stochastic volatility models with an application to daily stock returns, Unpublished paper, Department of Economics, Tokyo Metropolitan University.

[30] White, H. (1982). Maximum likelihood estimation of misspecified models, Econometrica, 50, 1-25. 